\title{
Health impact of Mediterranean diets in food at work
}

\author{
Federico Leighton ${ }^{1, *}$, Gianna Polic ${ }^{1}$, Pablo Strobel ${ }^{1}$, Druso Pérez ${ }^{1}$, Carlos Martínez ${ }^{1}$, \\ Luis Vásquez ${ }^{1}$, Oscar Castillo ${ }^{1}$, Luis Villarroel ${ }^{2}$, Guadalupe Echeverría ${ }^{1}$, Inés Urquiaga ${ }^{1}$, \\ Diego Mezzano ${ }^{1}$ and Jaime Rozowski ${ }^{1}$ \\ ${ }^{1}$ Center for Molecular Nutrition and Chronic Diseases, Pontificia Universidad Católica de Chile, Casilla 114-D, \\ Santiago, Chile: ${ }^{2}$ Public Health Department, Pontificia Universidad Católica de Chile, Casilla 114-D, \\ Santiago, Chile
}

Submitted December 2008: Accepted April 2009

\begin{abstract}
Objective: To evaluate the feasibility of diet mediterranisation, in a food-at-work context, and its consequence on metabolic syndrome in a mid-age unselected healthy male population group.

Design: One-year longitudinal intervention study. Physical exercise was not modified.

Setting: All workers of the Santiago division of 'Maestranza Diesel', a metalmechanic company servicing the mining industry, were invited to participate. Subjects: Initially, 145 workers of a total of 171, of average age 39 years, accepted to participate (sixteen women and 129 men). A subgroup of ninety-six men fully completed the controls programmed for the intervention study. Losses from the original group correspond to missing one control (sixteen), leaving the company (eleven) or blood sampling discomfort (six). The women and sixteen male workers, hired post study initiation, did participate but were excluded from this 12-month analysis.

Results: Diet mediterranisation was successful, reflected in the daily food consumption at the canteen and the evolution of the Mediterranean diet score (MDS) from $4 \cdot 8 \pm 1 \cdot 4$ to $7 \cdot 4 \pm 1 \cdot 5$ (limits $0-14$ ). Some metabolic syndrome components showed statistically significant improvement and also statistically significant correlation with the MDS: waist circumference, HDL-cholesterol, systolic and diastolic blood pressure. After 12 months, the reversion rate for metabolic syndrome was $48 \%(12 / 23)$ with an incidence rate of $4 \cdot 1 \%$ for new cases $(3 / 73)$. In total, metabolic syndrome decreased from $24 \cdot 0 \%$ to $15 \cdot 6 \%(23 / 96$ to $15 / 96)$ $(P=0 \cdot 029)$.

Conclusions: Diet mediterranisation is feasible in a food-at-work intervention, affecting lunch consumption at the workers canteen and overall consumption evaluated with MDS, together with a significant reduction in metabolic syndrome.
\end{abstract}

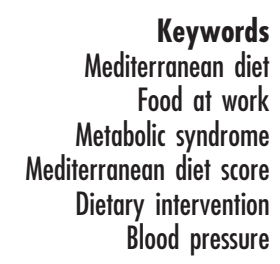

words

Food at work syndrome Dietary intervention Blood pressure
Mediterranean diets apparently are effective for the control of chronic diseases and also, for the control of metabolic syndrome, a condition associated to high risk of cardiovascular and all-causes death. Several studies, mostly made on Mediterranean populations, have used dietary scores to measure adherence to Mediterranean diet and have reported an inverse association with overall mortality $^{(1-8)}$. In one of these studies, 182 elder residents of three rural Greek villages were followed for approximately 5 years $^{(1)}$. The diet was assessed with a validated semi-quantitative questionnaire on food intake with a score built in terms of eight characteristic components. The results showed that a one-unit increase in diet score was associated with a significant $17 \%$ reduction in overall mortality.
The relationship between Mediterranean diet and metabolic syndrome has been investigated in several studies. Fish and omega-3 fatty acids, principal components of the Mediterranean diet, have been associated with a lower risk of cardiovascular disease ${ }^{(9)}$. Mediterranean diet improves blood pressure and lipid profile, decreases risk of thrombosis, improves endothelial function and insulin resistance, and reduces plasma homocysteine concentrations ${ }^{(10-15)}$. In 848 patients with first event of an acute coronary syndrome and 1078 persons without evidence of cardiovascular risk, from all Greek areas, Pitsavos et al. ${ }^{(16)}$ studied subjects with metabolic syndrome and observed that the Mediterranean diet was associated with a $35 \%$ reduction in coronary risk. 
In the classic Lyon Diet heart study, Renaud et al. ${ }^{(17)}$ evaluated the effect of a 'Cretan Mediterranean' diet on coronary heart disease, a study that gave great emphasis to the role of monounsaturated fatty acids (MUFA) in the protective effects of Mediterranean diets. In a pioneering intervention study, Esposito et al. ${ }^{(18)}$ studied patients with the metabolic syndrome, to assess the effect of a Mediterranean diet on endothelial function, vascular inflammatory markers and persistence of metabolic syndrome components. After 2 years of intervention, only $44 \%$ of the patients in the Mediterranean diet group still had the metabolic syndrome, while the patients in the control group did not show a significant reduction.

Preliminary results of another intervention study (PREDIMED) in Spain ${ }^{(19)}$ showed after 3 months that two variants of Mediterranean diet supplemented either with olive oil or nuts, produced beneficial changes in most risk-factor outcomes measured. Compared with a low-fat control diet, changes were found in plasma glucose levels, in blood pressure, in HDL-cholesterol and cholesterol/HDL-cholesterol ratio. The conclusion was that Mediterranean diets supplemented either with olive oil or nuts, are better than a low-fat diet to reduce metabolic syndrome.

In a 'food-at-work' innovative approach, we followed for 12 months the workers of a small industry in which a Mediterranean diet intervention of the canteen was performed, together with an educational program with focus on metabolic syndrome. Our objective was to evaluate the effects of diet mediterranisation on metabolic syndrome in a representative sample of Chilean workers.

\section{Subjects and methods}

\section{Study design}

A Mediterranean diet intervention study was carried out in a heavy machinery maintenance and repair company for the mining industry, 'Maestranza Diesel', in Santiago, Chile. All the employees or workers were invited to participate and were informed about Mediterranean diets and health, and on the relevance of the metabolic syndrome as a risk condition for chronic diseases. Initially, 145 workers from a total of 171 agreed to participate or were free of exclusion criteria. The group included the CEO and owner, other administrative authorities, office workers, technical personnel and specialised workers, a total of 129 men and sixteen women, with an average age of 39 years. The industry owners and administrators, the local workers union and the canteen contractor previously agreed to collaborate for the intervention.

During the 12-month observation period, there was some personnel turnover, some workers left the company, others were incorporated and some were temporally absent from Santiago at the moment of the controls. Thus, the complete follow-up was performed in a group of ninety-six male workers. Women and latecomers were studied but are not analysed in this communication. All workers were exposed to the educational activities of the project and to the canteen, free to choose offer modifications. The canteen contractor followed specific instructions for diet mediterranisation, while keeping traditional food alternatives for the small group of workers that declined participation. During the study, the volunteers were offered Mediterranean diet as the main canteen option.

Participants had a clinical, nutritional, anthropometric and laboratory evaluation immediately after being selected and every 4 months, for a full year.

\section{Inclusion criteria}

Maestranza Diesel workers who accepted to participate in the study, signed the informed consent form, and had lunch $5 \mathrm{~d}$ a week at the industry canteen.

\section{Exclusion criteria}

Persons under treatment for diabetes mellitus, blood hypertension and dyslipidaemia. Also, persons that actively adhered to specific diets or had participated in a weight loss program in the previous 6 months; and persons undergoing pharmacological treatment with drugs that modify lipid profiles, blood pressure, carbohydrate metabolism, plasma antioxidant capacity and inflammation.

\section{Cardiovascular risk factors and metabolic syndrome evaluation}

The diagnosis of the metabolic syndrome followed the Adult Treatment Panel III criteria: (i) abdominal adiposity (defined as waist circumference $>102 \mathrm{~cm}$ (men) or $>88 \mathrm{~cm}$ (women)); (ii) low levels of serum high-density lipoprotein cholesterol $(<40 \mathrm{mg} / \mathrm{dl}(\mathrm{men})$ or $<50 \mathrm{mg} / \mathrm{dl}$ (women)); (iii) hypertriglyceridaemia, $150 \mathrm{mg} / \mathrm{dl}$ or more; (iv) elevated blood pressure, $130 / 85 \mathrm{mmHg}$ or more; and (v) impaired glucose homeostasis (fasting plasma glucose concentration of $100 \mathrm{mg} / \mathrm{dl}$ or more $)^{(20)}$. The study was approved by the Ethics Committee of the Faculty of Medicine at the Catholic University of Chile.

\section{Mediterranean diet as the main canteen option for lunch}

The main characteristics of the food offer that the workers received during the intervention period were the following: a salad bar - previously not existent - presented everyday four different mixed salads, gastronomically attractive, plus two options for main dish and natural fruits as a dessert. To encourage salad consumption, an attractive olive oil-based salad dressing containing herbs and spices was continuously available. In addition to the daily Mediterranean diet menu, a vegetarian dish was available plus an option of beef with rice for consumers not interested in adhering to Mediterranean diet. Canola oil was routinely used with the exception of a 3-week period in months 7 and 8, when soy or sunflower oil was 
used for lack of canola supply. An olive oil bottle was routinely available at the salad bar counter; it helped to reinforce the concept of Mediterranean diet.

The volunteers were informed in small group talks, in their working time, about the objectives of the study, the concept of healthy life style, the advantages of consuming Mediterranean diet for the prevention of chronic diseases, the risk involved in metabolic syndrome, and received recommendations and specially prepared publications for the application of Mediterranean diet in their household. At least four times during the intervention period the workers attended educational talks. Among the recommendations were the following: for breakfast, a light meal consisting of tea or coffee with skimmed milk, yoghurt, whole wheat bread, fresh cheese, marmalade and avocado. For snacks, fruit, yoghurt and whole wheat bread with fresh cheese or low-fat cheese. For dinner, they were requested to eat Mediterranean dishes.

\section{Food at work record}

There was a daily qualitative and quantitative record of the food ingredients utilised at the canteen kitchen, a procedure requiring trained $a d-b o c$ personnel. These food records plus the number of people served were used to calculate average consumption by item, expressed as gram per person. Items were, basically, vegetables, fruits, potatoes, cereals, white wheat bread, whole wheat bread, red meat, white meat, legumes, fish and shellfish, vegetable oil, canola and olive oil.

Food record was done weighing the raw product, discounting the waste material in the preparation plus major leftovers, by item. The difference in weight between cooked and raw products was considered. White meat was poultry, chicken and lean pork. Red meat was beef. Dairy products included milk, yoghurt, ice cream, fresh cheese and other cheeses. At baseline, poultry, chicken, beef and pork with fat and/or skin, as well as whole milk were routinely used. During the study, poultry, chicken, beef and pork without fat and/or skin, and low-fat milk were employed. The vegetable fat item included sunflower or soy vegetable oil, and vegetable oil-based margarine and mayonnaise. Average consumption records were displayed at the canteen to keep the workers informed and motivated.

Diet composition was calculated employing the Food Processor II computer program (ESHA Research, Salem, OR, USA).

\section{Periodic controls of the volunteers and nutritional recommendations for diet mediterranisation}

The intervention started in June 2006 with a basal evaluation of each volunteer, and thereon, periodical controls were performed every 4 months. For each control, there was a data or sample collection interview, followed approximately 3 weeks later by a new personal interview to communicate results and for eventual medical and nutritional advice. The controls covered four aspects: clinical evaluation, anthropometric measurements, blood samples for clinical and biochemical measurements and nutritional interview. The nutritional evaluation consisted in a nutritionist-applied short questionnaire for the Mediterranean diet score (MDS) developed for the present study, plus forms and instructions for a $3 \mathrm{~d}$ weighed-food record to be completed at home.

At each control, personalised nutritional counselling was given on the basis of the food questionnaire records and cardiovascular risk factors evaluation. The average macronutrient composition of the Mediterranean diet recommended, supported by printed instructions, was as follows: carbohydrates, $50 \%$ to $60 \%$; proteins, $15 \%$ to $20 \%$; total fat, less than $30 \%$; saturated fat, less than $10 \%$; and cholesterol less than $300 \mathrm{mg}$ per day. The volunteers were advised to consume at least 300 to $350 \mathrm{~g}$ of vegetables, and 125 to $150 \mathrm{~g}$ of fruits per day; they were encouraged to consume whole grains (legumes, rice, corn and wheat) daily and to increase their consumption of olive oil. The fundaments of restriction in red meat consumption were given. Compliance with the program was assessed by attendance to the meetings, the programmed controls every 4 months and through completion of the questionnaires.

\section{Mediterranean diet score}

We elaborated a questionnaire with fourteen items to measure adherence to Mediterranean diet in Chile. It was designed on the basis of traditional food consumption habits in the European Mediterranean region with modifications to incorporate Chilean dietary habits. It was applied by nutritionists, and its purpose was to provide a simple overall measure of diet mediterranisation considering food at work, plus food during the rest of the day and during holidays. The components and consumption levels for the MDS applied are shown in Table 1.

Postulated beneficial components are: vegetables (without potatoes); legumes and nuts; fruits; whole grain cereals; lean meat; fish and shellish; low-fat and fermented dairy products; vegetable oils; olive and canola oils; avocado; and moderate wine consumption, ideally with meals. Components considered detrimental are: fatty meat and processed meat; full-fat dairy products not fermented; sugar; and excessive wine consumption, frequently not with meals, or lack of consumption.

A partial score value of $0, \frac{1}{2}$ or 1 was assigned to each item, considering usual consumption levels and its beneficial or detrimental quality. Olive and canola oils and avocado are separated items since in Chile, olive and canola oils are important sources of MUFA, and more so is avocado. This MDS ranges from 0 (minimal adherence) to 14 (maximal adherence).

\section{Analytical procedures}

Venous blood samples were collected at month 0 (before the intervention started) and after 4, 8 and 12 months of intervention. A $12 \mathrm{~h}$ fasting period preceded the collection 
Table 1 Mediterranean diet score definition

\begin{tabular}{|c|c|c|c|c|c|}
\hline & Score item & Score units & $1 \cdot 0$ points & 0.5 points & 0.0 points \\
\hline 1. & Vegetables (without potatoes) & Servings per day & $\geq 3$ & $1-3$ & $<1$ \\
\hline 2. & Legumes and nuts & Servings per week & $\geq 2$ & $1-2$ & $<1$ \\
\hline 3. & Fruits & Servings per day & $>2$ & $1-2$ & $<1$ \\
\hline 4. & Whole grain cereals & Servings per day & $\geq 2$ & $1-2$ & $<1$ \\
\hline 5. & Lean meat & Servings per week & $>4$ & $2-4$ & $<2$ \\
\hline 6. & Fish and shellfish & Servings per week & $>2$ & $1-2$ & $<1$ \\
\hline 7. & Fatty meat and processed meat & Servings per week & $<1$ & $1-2$ & $>2$ \\
\hline 8. & Full fat dairy products not fermented & Servings per day & $<1$ & $1-2$ & $\geq 2$ \\
\hline 9. & Low fat and fermented dairy products & Servings per day & $\geq 2$ & $1-2$ & $<1$ \\
\hline 10. & Vegetable oils & Teaspoons per day & $4-8$ & $2-4$ & $>8$ or $<2$ \\
\hline 11. & Olive and canola oil & Teaspoons per day & $>3$ & $1-3$ & $<1$ \\
\hline 12. & Avocado & Units per week & $>3$ & $0 \cdot 5-3$ & $<0.5$ \\
\hline 13. & Sugar & Teaspoons per day & $<4$ & $4-8$ & $>8$ \\
\hline 14. & Wine & Glasses per day & $\begin{array}{c}\text { Women: } \leq 1 \\
\text { Men: } \leq 2 \\
\text { Moderate and usually } \\
\text { with meals }\end{array}$ & $\begin{array}{c}\text { Women: } \leq 1 \\
\text { Men: } \leq 2 \\
\text { Moderate and rarely } \\
\text { with meals }\end{array}$ & $\begin{array}{c}\text { Women: }>1 \\
\text { Men: }>2 \\
\text { or non-drinkers }\end{array}$ \\
\hline
\end{tabular}

of samples into heparin, citrate and anticoagulant-free BD Vacutainer ${ }^{\circledR}$ tubes (Becton, Dickinson and Company, Franklin Lakes, NJ, USA). Plasma, serum, erythrocytes and leukocyte samples were stored, following common laboratory procedures until they were analysed. Plasma was obtained from freshly drawn blood by centrifugation at $1000 \mathrm{~g}$ for $5 \mathrm{~min}$.

Glucose, albumin, uric acid, bilirubin, creatinine, calcium, total cholesterol, HDL-cholesterol, LDL cholesterol, triglycerides, serum glutamic-oxaloacetic transaminase, gammaglutamyl transpeptidase and alkaline phosphatase were measured in serum using a spectrophotometer auto analyzer (Hitachi 917; Roche Diagnostics ${ }^{\circledR}$, Branchburg, NJ, USA) with reagent kits purchased from the manufacturer.

\section{Statistical analysis}

One-way ANOVA was used to compare daily consumption values, at lunch time, for each food item at baseline, 4, 8 and 12 months. One-way ANOVA for repeated measures was used to compare anthropometric, biochemical and clinical variables at baseline, 4, 8 and 12 months, in the subgroup of ninety-six male workers who participated for the entire 12 -month period. McNemar's $\chi^{2}$ test was used to compare metabolic syndrome prevalence in the same subgroup of ninety-six workers. Student's $t$ test for paired samples was used to compare the changes in MDS between subsequent control periods in the subgroup of ninety-six workers. A linear mixed-effects model analysis was used for correlation between MDS and metabolic syndrome components, in the same subgroup of workers.

\section{Results}

\section{Full participation group definition}

One hundred and forty-five workers of the Santiago division of 'Maestranza Diesel', 129 men and sixteen women, with an average age of 39 years, began the study in June
2006. The group represented approximately $85 \%$ of the workers of the Santiago division of the company; among them $24 \%$ had metabolic syndrome, and more specifically, following metabolic syndrome component definitions, $28 \%$ had abdominal obesity, $42 \%$ had high triglycerides, $28 \%$ had low HDL-cholesterol, 61\% had high blood pressure and $8 \%$ had high glucose. A subgroup of ninetysix male participants complied with the four programmed controls, the other thirty-three were not included since sixteen missed one control, six quit the study because they disliked the blood sampling procedure and eleven left the company. In this subgroup of ninety-six male workers, $24 \%$ had metabolic syndrome, 26\% abdominal obesity, 45\% high triglycerides, 26\% low HDL-cholesterol, 64\% high blood pressure and $7 \%$ high glucose. Thirty-seven per cent were smokers with average consumption of $4 \cdot 2$ units per day. The women participated in all controls, reinforcing the Maestranza Diesel worker's involvement in the project, yet their number was not enough for statistical evaluation. New workers (sixteen) hired along the study were also incorporated into the study for the same reason, yet they also were not included in the present analysis.

\section{Changes in food intake at work}

The evolution of food intake at lunch (canteen) is shown in Table 2; the data corresponds to the average daily consumption values in each one of the 4 months in which controls were performed. Diet mediterranisation is clearly observed. The comparison among the basal and 12th month food intake, shows increased consumption of vegetables, $51 \%$; fruits, $125 \%$; white meat, 29\%; fish and shellfish, 651\%; and cereals, 37\%; also with a 63\% decrease in red meat consumption. White bread was replaced by whole bread in about $50 \%$, but both were freely available. Potatoes, legumes and sugar consumption values remained constant. Canola oil was introduced at month 2 and was kept during the entire intervention with the exception of some supply restrictions during 
Table 2 Evolution of food composition at lunch time, average daily consumption per person at the canteen, along the 12-month diet mediterranisation intervention period

\begin{tabular}{|c|c|c|c|c|c|}
\hline Food item at lunch (grams/person) & Basal & Month 4 & Month 8 & Month 12 & $P$ value* \\
\hline Vegetables (without potatoes) & $175 \pm 27$ & $280 \pm 19$ & $330 \pm 63$ & $264 \pm 19$ & 0.001 \\
\hline Potatoes & $87 \pm 36$ & $83 \pm 50$ & $56 \pm 32$ & $83 \pm 26$ & 0.623 \\
\hline Fruits & $47 \pm 21$ & $93 \pm 30$ & $149 \pm 5$ & $106 \pm 13$ & 0.000 \\
\hline White meat & $53 \pm 27$ & $43 \pm 28$ & $56 \pm 25$ & $69 \pm 16$ & 0.535 \\
\hline Red meat & $104 \pm 19$ & $38 \pm 13$ & $32 \pm 4$ & $38 \pm 13$ & 0.000 \\
\hline Fish and shellfish & $3 \pm 1$ & $26 \pm 9$ & $17 \pm 8$ & $21 \pm 2$ & 0.001 \\
\hline Legumes & $13 \pm 4$ & $16 \pm 6$ & $16 \pm 2$ & $14 \pm 4$ & 0.622 \\
\hline Dairy products & $20 \pm 8$ & $7 \pm 7$ & $9 \pm 6$ & $11 \pm 5$ & 0.075 \\
\hline White bread & $80 \pm 9$ & $31 \pm 12$ & $34 \pm 5$ & $34 \pm 3$ & 0.000 \\
\hline Whole grain bread & 0 & $31 \pm 6$ & $24 \pm 5$ & $33 \pm 2$ & 0.000 \\
\hline Cereals & $45 \pm 8$ & $57 \pm 11$ & $49 \pm 3$ & $62 \pm 15$ & $0 \cdot 154$ \\
\hline Vegetable fat & $15 \pm 9$ & 0 & $13 \pm 10$ & $0 \pm 1$ & 0.011 \\
\hline Olive oil & 0 & $7 \pm 0$ & $7 \pm 1$ & $6 \pm 0$ & 0.000 \\
\hline Canola oil & 0 & $21 \pm 0$ & $9 \pm 11$ & $21 \pm 3$ & 0.000 \\
\hline Eggs & $12 \pm 6$ & $6 \pm 4$ & $6 \pm 2$ & $4 \pm 3$ & 0.058 \\
\hline Sugar & $16 \pm 4$ & $8 \pm 4$ & $14 \pm 1$ & $18 \pm 3$ & 0.005 \\
\hline MUFA/SFA & $1 \cdot 1 \pm 0 \cdot 0$ & $3 \cdot 7 \pm 0 \cdot 1$ & $3 \cdot 1 \pm 1 \cdot 1$ & $3 \cdot 2 \pm 0 \cdot 4$ & 0.000 \\
\hline Omega-6/omega-3 & $39 \cdot 3 \pm 6 \cdot 3$ & $2 \cdot 6 \pm 0 \cdot 2$ & $15 \cdot 4 \pm 11 \cdot 9$ & $2 \cdot 7 \pm 0 \cdot 1$ & 0.000 \\
\hline
\end{tabular}

MUFA, monounsaturated fatty acid; SFA, saturated fatty acid.

Data include all the workers that had lunch at the canteen, an average of $140,145,132,155$ workers per day at the basal month characterisation period and at months 4,8 and 12 , respectively.

*One-way ANOVA of daily consumption values for each food item.

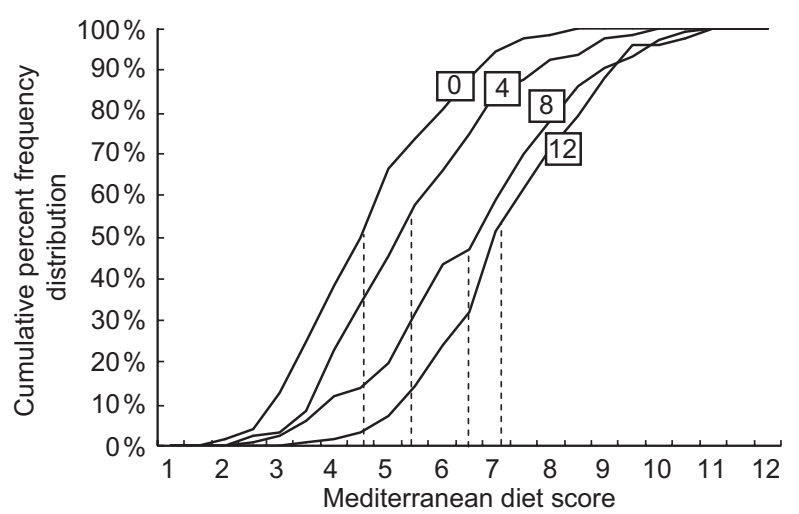

Fig. 1 Evolution of the Mediterranean diet score (MDS) values in the workers along the study. Results are expressed as cumulative per cent frequency distribution. Mean values for the MDS are shown in dotted lines

month 8; it was employed as cooking oil and as salad oil together with olive oil.

\section{Mediterranean diet score evolution}

The MDS was applied at each one of the four control points. The score values evolution, shown as cumulative per cent frequency distribution in Fig. 1, illustrates the growing mediterranisation of the diet along the 12-month intervention. The median value for the score distributions can be deduced from the figure and are very close to the average (and SD) score values $4 \cdot 8 \pm 1 \cdot 4,5 \cdot 6 \pm 1 \cdot 6$, $6 \cdot 8 \pm 1 \cdot 9$ and $7 \cdot 4 \pm 1 \cdot 5$, at baseline, 4,8 and 12 months, respectively. The score values frequency showed normal distribution at the four time points and the differences among subsequent mean values were statistically significant for each time interval.

\section{Changes in specific components and in the prevalence plus incidence of metabolic syndrome}

The intervention had no effect on body weight or BMI (Table 3). Among metabolic syndrome components, statistically significant changes were observed in values for waist circumference, HDL-cholesterol and for blood pressure. Blood glucose and plasma triglyceride values showed minor, not statistically significant, decreases (Table 3). Waist circumference decreased consistently and significantly along the study. HDL-cholesterol increased until month 8 but in month 12 it declined to basal level, but on the whole, the rise was significant. The most striking effect was observed in blood pressure; diastolic and systolic arterial blood pressure values declined consistently and significantly during the entire intervention, a drop in 15 and $13 \mathrm{~mm}$, respectively, along the study.

In the group of ninety-six male volunteer workers that participated for the entire 12 months, we observed an increase from $18 \%$ to $35 \%$ of volunteers free of any of the five components that define metabolic syndrome, while the group with three or more altered components, i.e. with metabolic syndrome, decreased from $24 \%$ to $16 \%$. As shown in Fig. 2, there was an apparent continuous decrease in the total number of metabolic syndrome cases, with a statistically significant decrease at the end of the study, a $35 \%$ decrease in metabolic syndrome volunteers (McNemar's $\chi^{2}$ test; $P=0 \cdot 029$ ).

\section{Correlation among changes in metabolic syndrome component values and Mediterranean diet score values}

The application of a linear mixed-effects model analysis allowed us to correlate the changes in MDS values with 
Table 3 Evolution of weight and metabolic syndrome parameters along the 12-month intervention period $(n$ 96)

\begin{tabular}{|c|c|c|c|c|c|}
\hline Variable & Basal & Month 4 & Month 8 & Month 12 & $P$ value* \\
\hline Body weight (kg) & $79 \cdot 5 \pm 10 \cdot 9$ & $79 \cdot 0 \pm 11 \cdot 0$ & $79 \cdot 0 \pm 11 \cdot 0$ & $79 \cdot 3 \pm 11 \cdot 1$ & 0.332 \\
\hline BMI $\left(\mathrm{kg} / \mathrm{m}^{2}\right)$ & $27 \cdot 8 \pm 3 \cdot 4$ & $27 \cdot 6 \pm 3 \cdot 3$ & $27 \cdot 6 \pm 3 \cdot 3$ & $27 \cdot 7 \pm 3 \cdot 3$ & 0.264 \\
\hline Waist circumference $(\mathrm{cm})$ & $96 \cdot 9 \pm 9 \cdot 3$ & $95 \cdot 7 \pm 8 \cdot 9$ & $95 \cdot 5 \pm 8 \cdot 9$ & $95 \cdot 2 \pm 8 \cdot 6$ & 0.002 \\
\hline Glucose $(\mathrm{mg} / \mathrm{dl})$ & $89 \cdot 8 \pm 21 \cdot 5$ & $89 \cdot 7 \pm 11 \cdot 6$ & $89 \cdot 6 \pm 18 \cdot 9$ & $88 \cdot 9 \pm 18 \cdot 4$ & $0 \cdot 826$ \\
\hline HDL cholesterol (mg/dl) & $45 \cdot 5 \pm 10 \cdot 5$ & $46 \cdot 0 \pm 10 \cdot 2$ & $47 \cdot 7 \pm 10 \cdot 7$ & $45 \cdot 4 \pm 10 \cdot 4$ & 0.000 \\
\hline Plasma triglycerides $(\mathrm{mg} / \mathrm{dl})$ & $159 \cdot 7 \pm 100 \cdot 7$ & $150 \cdot 7 \pm 125 \cdot 7$ & $156 \cdot 6 \pm 91 \cdot 4$ & $152 \cdot 5 \pm 84 \cdot 2$ & 0.628 \\
\hline Systolic blood pressure (mmHg) & $133 \cdot 4 \pm 18 \cdot 7$ & $126 \cdot 2 \pm 16 \cdot 1$ & $124 \cdot 2 \pm 10 \cdot 6$ & $120 \cdot 2 \pm 10 \cdot 6$ & 0.000 \\
\hline Diastolic blood pressure (mmHg) & $87 \cdot 4 \pm 10 \cdot 2$ & $83 \cdot 8 \pm 9 \cdot 9$ & $78 \cdot 4 \pm 8 \cdot 6$ & $72 \cdot 5 \pm 8 \cdot 3$ & 0.000 \\
\hline
\end{tabular}

${ }^{*}$ Repeated measurements variance analysis.

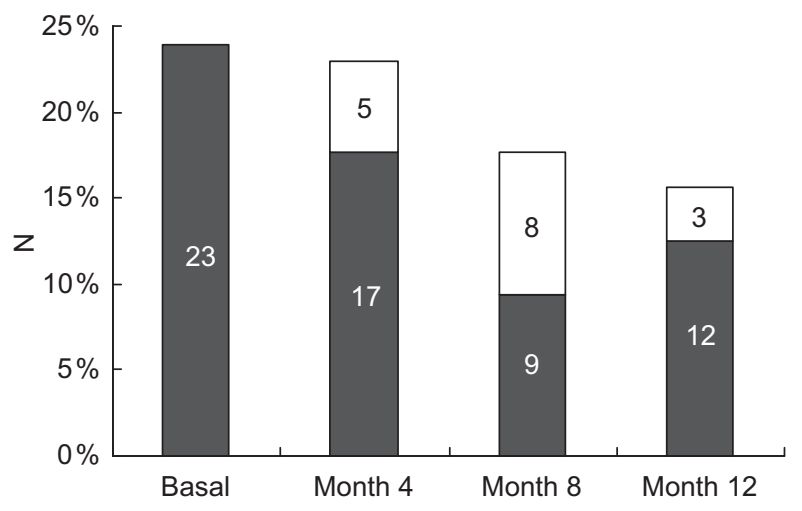

Fig. 2 Reversion of initial cases and incidence of new cases of metabolic syndrome along the 12-month intervention period (ninety-six workers). Figures in the bars identify number of cases. Metabolic syndrome cases detected at time 0 (black) plus new cases detected in subsequent controls (white)

the modification of individual metabolic syndrome component values, as shown in Table 4. We observed a significant positive correlation among increases in MDS and reduction in blood pressure and abdominal circumference, and also correlation among MDS values with elevation in HDL-cholesterol level (Table 4). No significant correlation was found among MDS and BMI, or plasma triglycerides or blood glucose.

\section{Discussion}

\section{Study design and participants}

The intervention made corresponds to a longitudinal study. Because of budget restrictions, we could not perform a parallel study in a control group of workers without intervention in their diet and educational-related activities.

In Chile, one-fifth of the formal workers, approximately 1200000 , receive food at the work place, predominantly lunch. The present study was done as a pilot with the purpose of evaluating the feasibility of Mediterranean diet food at work interventions and to measure its impact on the health of the workers. The experience obtained will be used to formulate a plan to scale-up food-at-work mediterranisation as a tool for the
Table 4 Mediterranean diet score correlation with metabolic syndrome components in volunteers $(n 96)$ that completed the 12-month intervention period

\begin{tabular}{lcc}
\hline & $\begin{array}{c}\text { Change per } \\
\text { Mediterranean } \\
\text { diet score unit }\end{array}$ & $P$ value \\
\hline BMI $\left(\mathrm{kg} / \mathrm{m}^{2}\right)$ & -0.04 & 0.136 \\
Waist circumference (cm) & -0.23 & 0.030 \\
Glucose $(\mathrm{mg} / \mathrm{dl})$ & -0.32 & 0.380 \\
HDL cholesterol (mg/dl) & +0.89 & 0.000 \\
Plasma triglycerides (mg/dl) & -3.51 & 0.210 \\
Systolic blood pressure $(\mathrm{mmHg})$ & -1.17 & 0.005 \\
Diastolic blood pressure $(\mathrm{mmHg})$ & -1.35 & 0.000 \\
\hline
\end{tabular}

*Linear mixed-effects model analysis.

prevention of chronic diseases in Chile, a project built on scientific evidences.

All workers, in fact all regular employees at 'Maestranza Diesel' were invited to participate and $85 \%$ accepted and complied with exclusion and inclusion criteria. A small number (six) of workers left the study because of blood sampling discomfort. All participants were interested in following the evolution of their food records, as well as changes in their clinical, anthropometric and biochemical measurements. The results presented correspond to a group of ninety-six male workers that completed all controls tests, but measurements were made in female workers and in those hired after the formal initiation of the study, basically because they felt that participation was a desirable opportunity. This attitude reflects that the concerted effort done by the owners and administrators, the workers union representatives and the research group members, was successful. The owners, administrators and workers union representatives fully participated as volunteers, which is an important educational factor. Additional professional support was received from chefs or other representatives from Dole ${ }^{\complement}$ and the industrial workers' safety organisation ACHS (Asociación Chilena de Seguridad). On the whole, this experimental setup explains achievements in food habits modifications: among others, the marked decrease in red meat consumption and the large increase in vegetables consumption; also, the shift to whole bread consumption and the incorporation of olive oil. The obvious question raised concerns the feasibility of a large scale-up of 
this pilot study and the strategic and logistic challenges involved.

\section{Mediterranean diet and metabolic syndrome}

The 2003 National Health Survey in Chile measured the prevalence of metabolic syndrome, ATPIII definition, and found that $23 \%$ of the population older than 17 years was affected, the same frequency for males and females ${ }^{(21)}$. The prevalence of metabolic syndrome in the workers from the present study was $24 \%$.

At the basal control point, metabolic syndrome components that were beyond the ATPIII cutoff point were observed in $60 \cdot 0 \%, 44 \cdot 4 \%, 26 \cdot 7 \%, 22 \cdot 2 \%$ and $7 \cdot 8 \%$ of the volunteers for hypertension, high triglycerides, low HDL-cholesterol, increased waist circumference and high blood glucose, respectively. The corresponding values after 12 months of intervention were $16 \cdot 7 \%$ and $34 \cdot 4 \%$ for hypertension and high triglycerides with a statistically significant decrease, while the presence of the other three components did not show a statistically significant variation. The most striking reduction is for hypertension. It is possible that 'Maestranza Diesel' workers might have had moderately elevated blood pressure, yet the average values found, shown in Table 3, for systolic and diastolic pressure were in between the values found for men aged 25-44 and 45-64 years in the National Health Survey ${ }^{(21)}$. In contrast to the $60 \%$ of volunteers with blood pressure above $130 / 85$, the National Health Survey published data shows that $40-45 \%$ of the male Chilean population would be above this cutoff point. The marked decrease in blood pressure observed suggests that further research is required to define the mechanisms involved. If it is a reproducible result for the mid-age male population in Chile, it would strongly justify the effort of scaling up food at work mediterranisation.

The results of the present study confirm other epidemiological and interventions studies that have showed a positive effect of Mediterranean diet on metabolic syndrome $^{(15,18,22-26)}$. The present study found a $35 \%$ of decrease in metabolic syndrome in the group of ninetysix workers. The reversion rate for the initial group with metabolic syndrome was $48 \%$ but there were three new cases at month 12 , a final reduction of $35 \%$ in metabolic syndrome cases.

Although, Mediterranean diet is inversely associated with BMI and obesity in epidemiological studies ${ }^{(8,24,27)}$, no change was observed in this group, a result that might relate to the age of the participants, the duration of the study or the small size of our group. From the $3 \mathrm{~d}$ weighed-food record (data not shown), we did not observe a change in caloric intake, at any of the control points, with an average caloric intake of 2068 (SD 560) (average and SD) for the ninety-six men group; furthermore the relative contribution of proteins, carbohydrates and fat, also did not change. This explains the lack of effect on weight and BMI.

\section{Possible mechanisms involved in the changes observed in metabolic syndrome components}

The largest change observed in this intervention was the reduction in blood pressure. It was equally observed in the group with metabolic syndrome, in the volunteers with one or two components of metabolic syndrome and in volunteers free of any metabolic syndrome component (data not shown). The DASH (Dietary Approaches to Stop Hypertension) trial demonstrated that a diet that emphasises fruits, vegetables and low-fat dairy products, that includes whole grains, poultry, fish and nuts, that contains only small amounts of red meat, sweets and sugar-containing beverages and that contains decreased amounts of total and saturated fat, lowers blood pressure substantially both in people with hypertension and those without hypertension, as compared with a typical diet in the United States ${ }^{(28,29)}$. Also, Psaltopoulou et al. ${ }^{(13)}$ have shown that adherence to Mediterranean diet was significantly and inversely associated with both systolic and diastolic blood pressure, the same for olive oil, vegetables, and fruit consumption, whereas cereals, meat and meat products, and ethanol intake were positively associated with arterial blood pressure. Mutual adjustment between olive oil and vegetables, which are frequently consumed together, indicated that olive oil had the dominant beneficial effect on arterial blood pressure in this population ${ }^{(13)}$. In the 'Maestranza Diesel' present intervention trial, MUFA/SFA (saturated fatty acid) ratio increased approximately three-fold along the intervention, basically because of a reduction in red and processed meat, the use of canola oil instead of vegetable oil and due to avocado consumption. Other studies have shown that a high intake of vegetables and whole grains associates with reduction in blood pressure ${ }^{(30)}$. Also, EPA and DHA can lower blood pressure as shown in human and animal studies; in a meta-analysis Morris et l. $^{(31)}$ found a significant reduction in blood pressure of 3.4/ $2.0 \mathrm{mmHg}$, in studies with hypertensive subjects who consumed $5.6 \mathrm{~g} / \mathrm{d}$ of omega-3 fatty acids. Likewise, Appel et al. ${ }^{(32)}$ found that blood pressure was decreased by $5 \cdot 5 /$ $3.5 \mathrm{mmHg}$ in trials of untreated hypertensive patients, who were given $3 \mathrm{~g} / \mathrm{d}$ of omega- 3 fatty acids. DHA seems to be more effective than EPA in lowering blood pressure $^{(33)}$. Still, in view of the high dose required to lower blood pressure and the proven efficacy of other nutritional factors and of antihypertensive medications, an increased intake of omega-3 fatty acids has a limited role in the management of hypertension ${ }^{(34)}$. In the present workers' intervention study, as shown in Table 2, the increase in fish consumption and in replacement of sunflower oil by canola oil, led to a large reduction in the omega-6/omega-3 ratio in food consumed at lunch time, a decrease from $39 \cdot 3$ to $2 \cdot 7$.

So the intervention diet rich in fruits, vegetables, lowfat dairy foods, with reduced saturated fat, with increased monounsaturated and omega- 3 fatty acids might be the 
main explanation for the blood pressure-lowering effect observed.

\section{Mediterranean diet score}

In order to quantify adherence to Mediterranean diet, a fourteen-item questionnaire adapted to Chilean food habits was designed. This food-frequency questionnaire, initially an experimental proposition, has been validated (thesis work to be published). The correlation of score values with metabolic syndrome component values, showed significant correlation with systolic and diastolic blood pressure, HDL-cholesterol and with waist circumference, but not with glucose or triglyceride levels. Blood glucose levels above $100 \mathrm{mg} / \mathrm{dl}$ were observed in $8 \%$ of the volunteers and the frequency was not modified with the dietary intervention, and with regard to fasting triglyceride level values, it is well known that they fluctuate and have a large coefficient of variation that renders statistical evaluation of the data difficult, so much so that recently it has been proposed the use of non-fasted triglyceride levels to evaluate cardiovascular risk ${ }^{(35)}$. The results obtained suggest that this MDS, based on fourteen food items, can be applied to measure adherence to Mediterranean diet and to estimate the risk of metabolic syndrome in Chile. It can also constitute a good tool in nutritional education and in the evaluation of the nutritional state in large groups

\section{Acknowledgements}

This study was supported by the Asociación Chilena de Seguridad (ACHS), DOLE Chile S.A. and the Pontificia Universidad Católica de Chile.

\section{References}

1. Trichopoulou A, Kouris-Blazos A, Wahlqvist ML, Gnardellis C, Lagiou P, Polychronopoulos E, Vassilakou T, Lipworth L \& Trichopoulos D (1995) Diet and overall survival in elderly people. BMJ 311, 1457-1460.

2. Trichopoulou A, Costacou T, Bamia C \& Trichopoulos D (2003) Adherence to a Mediterranean diet and survival in a Greek population. N Engl J Med 348, 2599-2608.

3. Trichopoulou A \& Critselis E (2004) Mediterranean diet and longevity. Eur J Cancer Prev 13, 453-456.

4. Knoops KT, de Groot LC, Kromhout D, Perrin AE, MoreirasVarela O, Menotti A \& van Staveren WA (2004) Mediterranean diet, lifestyle factors, and 10-year mortality in elderly European men and women: the HALE project. JAMA 292, 1433-1439.

5. Martinez-Gonzalez MA, Fernandez-Jarne E, Serrano-Martinez M, Wright M \& Gomez-Gracia E (2004) Development of a short dietary intake questionnaire for the quantitative estimation of adherence to a cardioprotective Mediterranean diet. Eur J Clin Nutr 58, 1550-1552.

6. Trichopoulou A, Orfanos P, Norat T et al. (2005) Modified Mediterranean diet and survival: EPIC-elderly prospective cohort study. BMJ 330, 991-997.

7. Bach A, Serra-Majem L, Carrasco JL, Roman B, Ngo J, Bertomeu I \& Obrador B (2006) The use of indexes evaluating the adherence to the Mediterranean diet in epidemiological studies: a review. Public Health Nutr 9 , 132-146.

8. Panagiotakos DB, Chrysohoou C, Pitsavos C \& Stefanadis C (2006) Association between the prevalence of obesity and adherence to the Mediterranean diet: the ATTICA study. Nutrition 22, 449-456.

9. Kris-Etherton PM, Harris WS \& Appel LJ (2002) Fish consumption, fish oil, omega-3 fatty acids, and cardiovascular disease. Circulation 106, 2747-2757.

10. Leighton F, Cuevas A, Guasch V et al. (1999) Plasma polyphenols and antioxidants, oxidative DNA damage and endothelial function in a diet and wine intervention study in humans. Drugs Exp Clin Res 25, 133-141.

11. Cuevas AM, Guasch V, Castillo O, Irribarra V, Mizon C, San Martin A, Strobel P, Perez D, Germain AM \& Leighton F (2000) A high-fat diet induces and red wine counteracts endothelial dysfunction in human volunteers. Lipids $\mathbf{3 5}$, 143-148.

12. Mezzano D, Leighton F, Martinez C et al. (2001) Complementary effects of Mediterranean diet and moderate red wine intake on haemostatic cardiovascular risk factors. Eur J Clin Nutr 55, 444-451.

13. Psaltopoulou T, Naska A, Orfanos P, Trichopoulos D, Mountokalakis T \& Trichopoulou A (2004) Olive oil, the Mediterranean diet, and arterial blood pressure: the Greek European Prospective Investigation into Cancer and Nutrition (EPIC) study. Am J Clin Nutr 80, 1012-1018.

14. Urquiaga I, Guasch V, Marshall G, San Martin A, Castillo O, Rozowski J \& Leighton F (2004) Effect of Mediterranean and Occidental diets, and red wine, on plasma fatty acids in humans. An intervention study. Biol Res 37, 253-261.

15. Panagiotakos DB \& Polychronopoulos E (2005) The role of Mediterranean diet in the epidemiology of metabolic syndrome; converting epidemiology to clinical practice. Lipids Health Dis 4, 7-12.

16. Pitsavos C, Panagiotakos DB, Chrysohoou C, Papaioannou I, Papadimitriou L, Tousoulis D, Stefanadis C \& Toutouzas P (2003) The adoption of Mediterranean diet attenuates the development of acute coronary syndromes in people with the metabolic syndrome. Nutr J 2, 1-7.

17. Renaud S, de Lorgeril M, Delaye J, Guidollet J, Jacquard F, Mamelle N, Martin JL, Monjaud I, Salen P \& Toubol P (1995) Cretan Mediterranean diet for prevention of coronary heart disease. Am J Clin Nutr 61, 1360S-1367S.

18. Esposito K, Marfella R, Ciotola M, Di Palo C, Giugliano F, Giugliano G, D'Armiento M, D'Andrea F \& Giugliano D (2004) Effect of a Mediterranean-style diet on endothelial dysfunction and markers of vascular inflammation in the metabolic syndrome: a randomized trial. JAMA 292, 1440-1446.

19. Estruch R, Martinez-Gonzalez MA, Corella D et al. (2006) Effects of a Mediterranean-style diet on cardiovascular risk factors: a randomized trial. Ann Intern Med 145, 1-11.

20. Grundy SM, Cleeman JI, Daniels SR et al. (2005) Diagnosis and management of the metabolic syndrome: an American Heart Association/National Heart, Lung, and Blood Institute Scientific Statement. Circulation 112, 2735-2752.

21. Ministerio de Salud (2003) Encuesta Nacional de Salud. Chile. http://epi.minsal.cl/epi/html/elvigia/VIGIA20.pdf

22. Serra-Majem L, Roman B \& Estruch R (2006) Scientific evidence of interventions using the Mediterranean diet: a systematic review. Nutr Rev 64, S27-S47.

23. Esposito K, Ciotola M \& Giugliano D (2007) Mediterranean diet and the metabolic syndrome. Mol Nutr Food Res 51, 1268-1274.

24. Schroder H, Marrugat J, Vila J, Covas MI \& Elosua R (2004) Adherence to the traditional Mediterranean diet is inversely associated with body mass index and obesity in a Spanish population. J Nutr 134, 3355-3361. 
25. Mendez MA, Popkin BM, Jakszyn P et al. (2006) Adherence to a Mediterranean diet is associated with reduced 3-year incidence of obesity. J Nutr 136, 2934-2938.

26. Salas-Salvado J, Fernandez-Ballart J, Ros E et al. (2008) Effect of a Mediterranean diet supplemented with nuts on metabolic syndrome status: one-year results of the PREDIMED randomized trial. Arch Intern Med $\mathbf{1 6 8}$, 2449-2458.

27. Panagiotakos DB, Pitsavos C, Skoumas Y, Lentzas Y, Papadimitriou L, Chrysohoou C \& Stefanadis C (2008) Abdominal obesity, blood glucose and apolipoprotein B levels are the best predictors of the incidence of hypercholesterolemia (2001-2006) among healthy adults: the ATTICA study. Lipids Health Dis 7, 11-18.

28. Appel LJ, Moore TJ, Obarzanek E et al. (1997) A clinical trial of the effects of dietary patterns on blood pressure. DASH Collaborative Research Group. $N$ Engl J Med 336, $1117-1124$

29. Sacks FM, Svetkey LP, Vollmer WM et al. (2001) Effects on blood pressure of reduced dietary sodium and the Dietary Approaches to Stop Hypertension (DASH) diet. DASH-Sodium Collaborative Research Group. N Engl J Med 344, 3-10.
30. Behall KM, Scholfield DJ \& Hallfrisch J (2006) Wholegrain diets reduce blood pressure in mildly hypercholesterolemic men and women. J Am Diet Assoc 106, $1445-1449$.

31. Morris MC, Sacks F \& Rosner B (1993) Does fish oil lower blood pressure? A meta-analysis of controlled trials. Circulation 88, 523-533.

32. Appel LJ, Miller ER 3rd, Seidler AJ \& Whelton PK (1993) Does supplementation of diet with 'fish oil' reduce blood pressure? A meta-analysis of controlled clinical trials. Arch Intern Med 153, 1429-1438.

33. Mori TA, Watts GF, Burke V, Hilme E, Puddey IB \& Beilin LJ (2000) Differential effects of eicosapentaenoic acid and docosahexaenoic acid on vascular reactivity of the forearm microcirculation in hyperlipidemic, overweight men. Circulation 102, 1264-1269.

34. Jung UJ, Torrejon C, Tighe AP \& Deckelbaum RJ (2008) n-3 Fatty acids and cardiovascular disease: mechanisms underlying beneficial effects. Am J Clin Nutr 87, 2003S-2009S.

35. Warnick GR \& Nakajima K (2008) Fasting versus nonfasting triglycerides: implications for laboratory measurements. Clin Chem 54, 14-16. 